\title{
Satellite magnetic resonances of a bound pair of half-quantum vortices in rotating superfluid ${ }^{3} \mathrm{He}-A$
}

\author{
Chia-Ren $\mathrm{Hu}$ \\ Center for Theoretical Physics, Department of Physics, Texas A\&M University, College Station, Texas 77843-4242 \\ Kazumi Maki \\ Department of Physics, University of Southern California, Los Angeles, California 90089-0484
}

(Received 29 December 1986)

\begin{abstract}
The transverse magnetic resonance satellite frequency and intensity associated with a bound pair of half quantum vortices in a thin slab of ${ }^{3} \mathrm{He}-A$, rotating about the slab normal and subject to a magnetic field applied at an angle $\theta$ with the slab normal, is recalculated in a variational approach. This approach takes properly into account the azimuthal symmetry of the spin wave function and therefore corrects a previous prediction by Salomaa and Volovik. The corresponding quantities for the longitudinal resonance are also calculated. In addition, a general criterion is given for determining the physical transverse-resonance wave function in a large magnetic field in the presence of any distribution of spin disgyrations of any kinds, whether with integer or fractional Frank indices.
\end{abstract}

\section{INTRODUCTION}

In a recent Letter, Salomaa and $\operatorname{Volovik}^{1}(\mathrm{SV})$ suggested that a rather novel type of superfluid vortex, with only a half quantum of circulation [i.e., $p=\frac{1}{2}$, where $\left.p \equiv(M / h) \oint \mathbf{v}_{s} \cdot d \mathbf{r}\right]$, is not only theoretically possible in the $A$-phase of superfluid ${ }^{3} \mathrm{He}$ as is deduced previously from topological considerations, ${ }^{2}$ but is in fact also experimentally observable in certain laboratory arrangements. They showed that such vortices become energetically most stable in such circumstances in comparison with the other types of vortices which are also predicted in topological considerations that are possible in ${ }^{3} \mathrm{He}-A$, i.e., the usual type of singular vortices with $p=1$, and the coreless type of vortices with $p=2$. The experimental setup they proposed to observe such "half-quantum vortices" is a thin slab of ${ }^{3} \mathrm{He}-A$, with a thickness $D \lesssim \xi_{D} \approx 10^{-3} \mathrm{~cm}$, the dipole coherence length, rotating about the slab normal $\widehat{\mathbf{N}} \| \widehat{\mathbf{z}}$ at an angular velocity $\Omega$, and with a uniform magnetic field $\mathbf{H}>50 \mathrm{G}$ applied either along $\hat{\mathbf{z}}$, or tilted from $\widehat{\mathbf{z}}$ by an angle $\theta$.

For $\theta$ not overly small they further showed that the half-quantum vortices will form bound pairs, with a characteristic satellite transverse-NMR signal which exhibits (1) an enormously large negative shift from the bulk signal; (2) a sensitive temperature dependence in its position and intensity; and (3) an intensity proportional to $\Omega$ and $\sin ^{-2} \theta$. This is because that, as is noted by SV, a half-quantum vortex must be accompanied by a singular texture of the spin axis $\hat{\mathrm{d}}$, characterized by a Frank index $m= \pm \frac{1}{2}$, meaning that $\hat{\mathbf{d}}$ must turn $\pm 180^{\circ}$, and therefore changes to $-\widehat{\mathbf{d}}$, as any closed path encircling the vortex axis is traversed. Such a $\widehat{d}$ distribution can be single valued, only if a two-dimensional "cut" extends from the vortex axis out to the sample boundary or to another vortex axis (with $m=\mp \frac{1}{2}$ ). Across such a cut, $\widehat{\mathbf{d}}$ must change sign and is therefore discontinuous. The complete order parameter

$$
\Delta_{\mu i}=\Delta_{0} e^{i \Phi}(\hat{\mathbf{m}}+i \widehat{\mathbf{n}})_{\mu} \widehat{\mathbf{d}}_{i},
$$

with constant $\Delta_{0}, \hat{\mathbf{m}}$, and $\hat{\mathbf{n}}=\widehat{\mathbf{z}} \times \hat{\mathbf{m}}$, but spatially dependent $\Phi$ and $\hat{\mathbf{d}}$, can nevertheless be nonsingular everywhere including on the cut if the phase factor $e^{i \Phi}$ also changes its sign across the same cut. This is, of course, achieved by having $p=\frac{1}{2}$ (or $-\frac{1}{2}$ ), corresponding to $\Phi$ changing continuously, say from 0 to $\pi$ (or $-\pi$ ), as the same closed curve around the vortex is traversed, starting and ending on the same cut.

For $\theta \neq 0$, the excess dipole energy associated with an isolated half-quantum vortex must be proportional to the volume of the sample. To keep this energy independent of the sample volume, a $\left(p=\frac{1}{2}, m=\frac{1}{2}\right)$ vortex must bind with a $\left(p=\frac{1}{2}, m=-\frac{1}{2}\right)$ vortex to form a bound pair. When the separation $R$ of such a vortex pair is large, a $\hat{\mathrm{d}}$ soliton or domain wall must stretch between the two vortex axes, giving a dipole energy growing linearly in $R$. On the other hand, for small $R$ the same mechanism which prevents two singly quantized vortices from coalescing into one doubly quantized one (in superfluid ${ }^{4} \mathrm{He}$ or in superconductors), i.e., the flow energy for the latter is essentially twice as large as that for the former, gives rise to an energy divergent as $\ln \left(r_{\Omega} / R\right)$. While this repulsive energy for the vortex pair is partially compensated by an attractive one due to the $\widehat{\mathbf{d}}$ gradient energy which has a singular term proportional to $\ln (R / \xi)(\xi$ being the size of the vortex cores), this does not prevent the existence of an equilibrium separation $R_{\min }$ between the two vortices in a bound pair which can be much smaller than the mean vortex distance $r_{\Omega} \sim(h / 4 M \Omega)^{1 / 2}$ if $\Omega$ is not too large. In this limit each bound vortex pair can be considered isolated, with asymptotically uni- 
form $\widehat{\mathbf{d}}$, giving rise to a bound spin-wave mode which $\mathrm{SV}$ studied.

In a subsequent comment, ${ }^{3}$ one of us (K.M.) pointed out that the enormously large negative NMR shift predicted by SV cannot be physically correct, and that the spin wave function for the physical transverse bound spin-wave mode must have the proper azimuthal symmetry as given by the phase factor $e^{ \pm i \phi / 2}$ about each halfquantum vortex axis, the reason offered being that otherwise the spin-wave mode would not couple to a uniform rf field. In a reply by $\mathrm{SV},{ }^{4}$ they agreed to the incorrectness of their previous magnetic resonance predictions for half-quantum vortex pairs, but noted a different reason, viz., their previously studied bound spin-wave mode is actually unphysical due to the topological observation that since the equilibrium $\widehat{\mathbf{d}}$ configuration is described by a double-valued function, the corresponding spin wave function for the transverse bound spin-wave mode must also be double valued. This point is not completely obvious since the spin wave function for the corresponding longitudinal mode actually remains single valued under such circumstances. We offer a simple intuitive explanation of this point as follows. If one connects the heads and tails, separately, of all $\hat{\mathbf{d}}(\mathbf{r})$ vectors located on a closed curve encircling the axis of a half-quantum vortex, one obtains a Möbius band flattened to lie in a plane. (See Fig. 1.) It can then be seen that to study the longitudinal and transverse bound NMR modes of the associated $\hat{\mathbf{d}}$ texture is essentially equivalent to the study of the in-plane and out-of-plane normal-mode vibrations of this flattened Möbius band, respectively. ${ }^{5}$ It is then not difficult to convince oneself that whereas the inplane oscillation can have the same amplitude everywhere along the Möbius band, the out-ofplane-oscillation amplitude must have at least one node and change sign in encircling the Möbius band, or else the Möbius band would be torn apart at some point.

As to what is the real justification that the spin wave function for the transverse magnetic resonance mode must have the proper azimuthal symmetry, and therefore the one found by SV in Ref. 1 must be incorrect, we

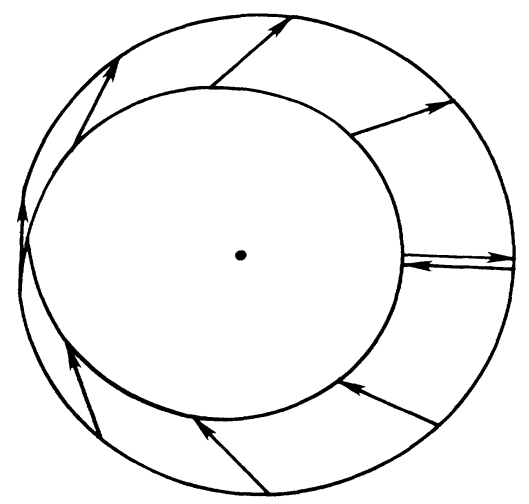

FIG. 1. If one connects the heads and tails, separately, of all $\hat{\mathbf{d}}(\mathbf{r})$ vectors located on a closed curve encircling the axis of a half-quantum vortex line, one obtains a Möbius band flattened to lie in a plane. find that it is neither the topological point of Ref. 4 alone, nor the coupling consideration of Ref. 3 alone, but one must always consider both simultaneously in order to obtain an unambiguous and general criterion for determining such a wave function for any texture containing singularities, except that in the case where no half-quantum vortices are involved the topological point leads only to a relatively trivial conclusion which is usually taken for granted.

The purpose of this paper is to clarify this point (in the Appendix), and to report our new results (using a variational approach) on the transverse magnetic resonance frequency and its intensity for the half-quantum vortex-pair texture, taking properly into account the point raised in Ref. 3. For completeness we have also studied the same quantities for the longitudinal magnetic resonance of the same texture. We shall see that NMR remains to be a promising way for detecting these novel objects, even though the transverse shifts are now found to be substantially smaller than those predicted by SV. The temperature dependences of these shifts and their intensities are also found to be completely different from the predictions in Ref. 1.

In Sec. II we present a variational calculation of the equilibrium texture associated with such a vortex pair, and the value of their equilibrium separation $R_{\text {min }}$ as determined within this approach. Both of these agree well with and extend beyond the more elaborate calculation by SV of the same quantities. In Sec. III the longitudinal and transverse NMR satellite frequencies and their corresponding intensities are calculated along $\mathbf{a}$ similar variational approach. However, a subtlety is revealed in the choice of the variational trial function for the calculation of the transverse satellite resonance frequency and its intensity due to the constraint on the proper azimuthal symmetry of the spin wave function. Thus in Sec. IV we have further analyzed the asymptotic behavior of the predicted quantities in order to further enhance our confidence in these results. Sec. $\mathrm{V}$ contains a short summary and conclusion and in the Appendix we will present a general and precise criterion for the determination of the physical solutions for the transverse magnetic resonances in the presence of any number of $\hat{\mathbf{d}}$ disgyration lines of any kinds, whether characterized by integer or half-odd-integer Frank indices; the only limitation being that the external magnetic field $\mathbf{H}$ has been assumed to be so large that $\widehat{\mathbf{d}}$ is everywhere confined to the plane perpendicular to $\mathbf{H}$.

\section{EQUILIBRIUM TEXTURE}

For the geometry considered by SV, the rotational axis $\widehat{\mathbf{\Omega}}$ and the anisotropy axis $\hat{\boldsymbol{l}}$ are both in the $z$ direction. An applied magnetic field is taken to be along the direction $\widehat{\mathbf{z}}^{\prime} \equiv \hat{\mathbf{z}} \cos \theta+\hat{\mathbf{x}} \sin \theta$. If we then define $\widehat{\mathbf{x}}^{\prime} \equiv-\widehat{\mathbf{z}} \sin \theta+\widehat{\mathbf{x}} \cos \theta$, we expect the dipole energy to align $\hat{\mathbf{d}}$ along $\pm \hat{\mathbf{x}}^{\prime}$ in the absence of any $\hat{\mathbf{d}}$ texture, and if the applied field $H$ is $>50 \mathrm{G}$, so that $\hat{\mathrm{d}}$ is everywhere forced to lie in the $x^{\prime} y$ plane by the dominant magnetic anisotropy energy. We can then describe any localized texture by taking $\mathbf{d}=-\widehat{\mathbf{x}}^{\prime} \cos \alpha+\hat{\mathbf{y}} \sin \alpha$ and 
expect $\alpha$ to approach zero (or $\pi$ ) outside the range of the localized texture. For a half-quantum vortex pair located at $x\left(\right.$ not $\left.x^{\prime}\right)= \pm R / 2$ we must have $\alpha=\alpha_{\text {sing }}+\alpha_{\text {reg }}$ with

$$
\begin{aligned}
\alpha_{\text {sing }} & \equiv \frac{1}{2}\left(\phi_{1}-\phi_{2}\right) \\
& =\frac{1}{2}\left(\tan ^{-1} \frac{y}{x-R / 2}-\tan ^{-1} \frac{y}{x+R / 2}\right),
\end{aligned}
$$

and $\alpha_{\text {reg }}$ a nonsingular function of $x$ and $y$. It is convenient to work with the coordinates

$$
\begin{aligned}
& x=\frac{R}{2} \cosh u \cos v, \\
& y=\frac{R}{2} \sinh u \sin v,
\end{aligned}
$$

then $\alpha_{\operatorname{sing}}=\tan ^{-1}(\sin v / \sinh u)$. For a simple variational representation of the texture we can therefore take

$$
\alpha=\tan ^{-1}[F(u) \sin v / \sinh u],
$$

and require $F(u) \rightarrow 1$ as $u \rightarrow 0$, and $\rightarrow 0$ as $u \rightarrow \infty$. We have worked with

$$
\begin{aligned}
& F_{1}(u) \equiv \exp [-a(\cosh u-1)], \\
& F_{2}(u) \equiv \exp (-a \sinh u),
\end{aligned}
$$

and will present both sets of results. Actually we have also tried some other more complex $F(u)$ without obtaining better success, and therefore will not report them.

The total free energy to be minimized, including the $\widehat{d}$-gradient energy and the dipole energy, is (per unit thickness along $z$ ):

$$
F=A \int d^{2} r\left[\left|\nabla_{\perp} \alpha\right|^{2}+\hat{\xi}_{D}^{-2} \sin ^{2} \alpha\right]
$$

where $\left|\nabla_{\perp} \alpha\right|^{2} \equiv(\partial \alpha / \partial x)^{2}+(\partial \alpha / \partial y)^{2}, \quad A \equiv \frac{1}{2}(\hbar / M)^{2} \rho_{s p}$ in the notations of Ref. 1 , and $\widetilde{\xi}_{D} \equiv \xi_{D} / \sin \theta$ has also been introduced in Ref. 1. Since

$$
d^{2} r=(R / 2)^{2}\left(\cosh ^{2} u-\cos ^{2} v\right) d u d v
$$

and

$$
d^{2} r\left|\nabla_{\perp} \alpha\right|^{2}=d u d v\left[(\partial \alpha / \partial u)^{2}+(\partial \alpha / \partial v)^{2}\right],
$$

it is clear that the optimum variational parameter $a$ and the reduced free energy $f \equiv F / A$ are both functions of $\bar{R} \equiv R / \widetilde{\xi}_{D}$. The minimization was performed numerically. (Notice that for the chosen trial functions the $v$ integrals can all be done analytically.) The results are shown in Fig. 2, where $\Delta f$ is $f$ minus an $a$-independent singular term $\pi \ln (R / \xi)$, with $\xi$ being the core size of the singular half-quantum vortices. The dashed lines correspond to the first trial function $F_{1}(u)$ of Eq. (5a), whereas the solid lines correspond to $F_{2}(u)$ of Eq. (5b). As shown in this figure, the trial function $F_{2}(u)$ gives lower $\Delta f$ and is a better trial function for essentially the whole range of $\bar{R}$. [Actually for $\bar{R} \leqslant 1.0$, we obtained a slightly lower energy from using $F_{1}(u)$, but the difference in $\Delta f$ is so small that it does not show in this plot.] Using the $\Delta f(\bar{R})$ determined with $F_{2}(u)$, we have then calculated the equilibrium separation of the two vortices in a bound pair $\bar{R}_{\text {min }}$ as a function of $\rho_{s p}^{\perp} / \rho_{s}^{1}$, following the procedure already outlined in Ref. 1 . The result is plotted in Fig. 3. For $\rho_{s p}^{\perp} / \rho_{s}^{\perp} \geq 0.45$ this result may be compared with Fig. 2(b) of Ref. 1. The largest difference occurs near $\rho_{s p}^{\perp} / \rho_{s}^{\perp} \cong 0.45$ where our value for $\bar{R}_{\text {min }}$ is smaller by about $5 \%$. This probably in part reflects the error in our variational determination of $\Delta f(\bar{R})$, although we do not know the accuracy in the calculation presented in Ref. 1. Anticipating the need in the later sections, we have calculated $\bar{R}_{\min }$ down to $\rho_{s p}^{\perp} / \rho_{s}^{\perp}=0.1$. We not that the value of $\rho_{s p}^{\perp} / \rho_{s}^{\perp}$ is a function of the pressure $P$ and the temperature $T$, and that according to the weak-coupling theory with Fermi-liquid corrections, ${ }^{6}$ the smallest value of $\rho_{s p}^{\perp} / \rho_{s}^{\perp}$ occurs at $T=0$ at the melting pressure, where it is 0.116 if the Fermiliquid parameters as determined by $\mathrm{Greywall}^{7}$ are used. On the other hand $\rho_{s p}^{\perp} / \rho_{s}^{\perp} \rightarrow 1$ as $T \rightarrow T_{c}$, so the range of $\rho_{s p}^{\perp} / \rho_{s}^{\perp}$ values we have studied should cover essentially the whole range of physical interest.

\section{MAGNETIC RESONANCES}

To study the magnetic resonances we let

$\widehat{\mathbf{d}}=\left[-\widehat{\mathbf{x}}^{\prime} \cos (\alpha+f)+\hat{\mathbf{y}} \sin (\alpha+f)\right] \cos g+\widehat{\mathbf{z}}^{\prime} \sin g$.

When the amplitudes of $f$ and $g$ are small, the longitudinal and transverse magnetic resonance modes correspond to $f \neq 0, g=0$ and $f=0, g \neq 0$, respectively. (Actually this is true only after neglecting a very weak coupling between the two modes due to their very different resonance frequencies, especially at high magnetic fields.)

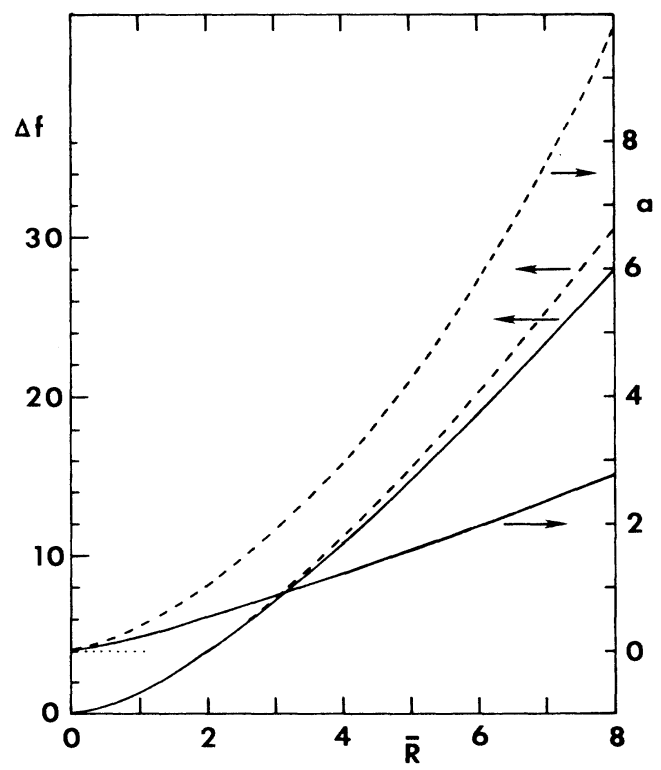

FIG. 2. Plotted are the excess free energy $\Delta f \equiv f$ $-\pi \ln (R / \xi)$ (the left scale) and the variational parameter $a$ in Eqs. (5a) and (5b) (the right scale) as functions of $\bar{R} \equiv R / \xi_{D}$. The solid lines are obtained with $F_{2}(u)$ of Eq. $(5 \mathrm{~b})$ as the trial function in Eq. (4), whereas the dashed lines are obtained with $F_{1}(u)$ of Eq. (5a). 


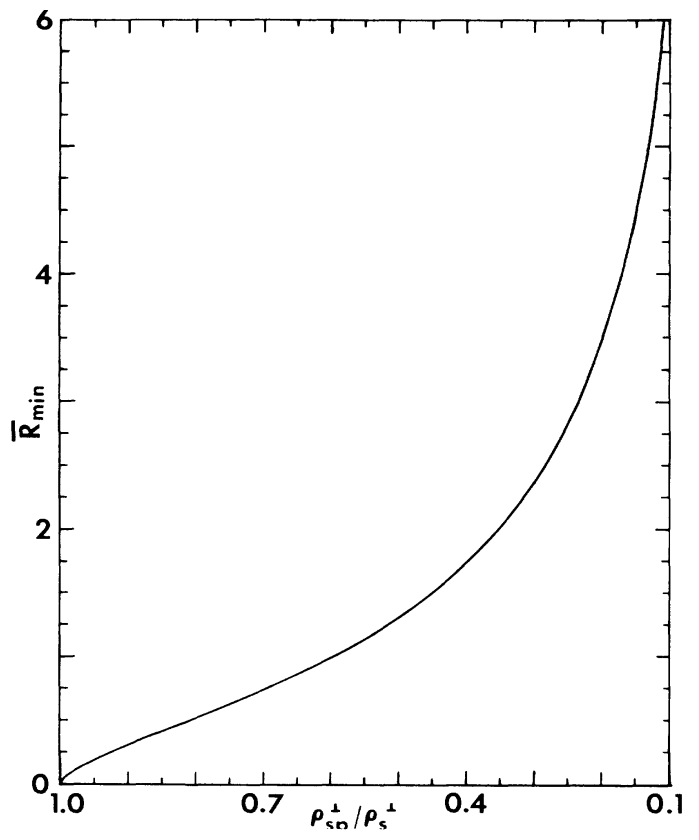

FIG. 3. Equilibrium intrapair separation $R_{\min }$ in units of $\widetilde{\xi}_{D}$ is plotted as a function of $\rho_{s p}^{\perp} / \rho_{s}^{\perp}$. This figure extends beyond Fig. 2(b) of Ref. 1.

Then the longitudinal and transverse resonance frequencies are given by, respectively,

$$
\begin{aligned}
& \omega_{l}^{2}=\Omega_{L}^{2}\left(1+\lambda_{f}\right) \sin ^{2} \theta, \\
& \omega_{t}^{2}=\gamma^{2} H^{2}+\Omega_{L}^{2}\left(-\cos 2 \theta+\lambda_{g} \sin ^{2} \theta\right),
\end{aligned}
$$

where $\Omega_{L}$ is the Leggett frequency, and $\lambda_{f}$ and $\lambda_{g}$ are obtained by solving

$$
\begin{aligned}
& -\widetilde{\xi}_{D}^{2} \nabla_{\perp}^{2} f-2 f \sin ^{2} \alpha=\lambda_{f} f, \\
& -\widetilde{\xi}_{D}^{2}\left(\nabla_{\perp}^{2}+\left|\nabla_{\perp} \alpha\right|^{2}\right) g-g \sin ^{2} \alpha=\lambda_{g} g .
\end{aligned}
$$

The intensities of these magnetic resonance modes (for a uniform driving $\mathrm{rf}$ field) are proportional to their respective oscillator strength:

$I_{f} \propto \frac{1}{2} n_{v}\left|\int f d^{2} r\right|^{2} / \int|f|^{2} d^{2} r \equiv \frac{1}{2} n_{v} \widetilde{\xi}_{D}^{2} \widetilde{I}_{f}$,

$I_{g} \propto \frac{1}{2} n_{v}\left|\int g e^{-i \alpha} d^{2} r\right|^{2} / \int|g|^{2} d^{2} r \equiv \frac{1}{2} n_{v} \widetilde{\xi}_{D}^{2} \widetilde{I}_{g}$,

where $n_{v}=2 M \Omega / \pi \hbar$ is the real density of half-quantum vortices. (This was given incorrectly in Ref. 1.)

In Ref. 3 (and 4) it was pointed out that the physical solution for Eq. (11) is a $g(\mathbf{r})$ which has a phase $e^{ \pm i \phi / 2}$ around the $m= \pm \frac{1}{2}$ disgyration, and therefore has a sign discontinuity across a surface stretching between the two vortex axes. In Ref. 3 it was proposed to achieve this by letting

$$
g(\mathbf{r})=e^{ \pm i \alpha(\mathbf{r})} \widetilde{g}(r),
$$

with $\mathbf{g}(\mathbf{r})$ a (single-valued, continuous) solution of the equation

$$
-\widetilde{\xi}_{D}^{2} \nabla_{\perp}^{2} \widetilde{g}-\widetilde{g} \sin ^{2} \alpha=\lambda_{g} \widetilde{\mathbf{g}} .
$$

Implicit in this statement is the assumption that $\widetilde{g}$ is real. We have now discovered that this assumption is not exactly true, and that there is a much better choice for the variational function than taking $\widetilde{g}$ real which gives the correct asymptotic value of $\lambda_{g}$ for $R \rightarrow \infty$. To see this we first convert Eqs. (10) and (11) to

$$
\begin{aligned}
& \lambda_{f}=\min _{f} \frac{\widetilde{\xi}_{D}^{2} \int\left|\nabla_{\perp} f\right|^{2} d^{2} r-2 \int\left(\sin ^{2} \alpha\right)|f|^{2} d^{2} r}{\int|f|^{2} d^{2} r} \\
& \lambda_{g}=\min _{g} \frac{\widetilde{\xi}_{D}^{2} \int\left(\left|\nabla_{\perp} g\right|^{2}-\left|\nabla_{\perp} \alpha\right|^{2}|g|^{2}\right) d^{2} r-\int\left(\sin ^{2} \alpha\right)|g|^{2} d^{2} r}{\int|g|^{2} d^{2} r} .
\end{aligned}
$$

As a trial wave function for $f$ one can simply take, for example,

$$
f_{1}(u)=\exp \left(-\frac{1}{2} C \cosh u\right) .
$$

However, we find that the expression

$$
f_{2}(u)=(\cosh u)^{-1 / 2} \exp \left(-\frac{1}{2} C \cosh u\right)
$$

gives a better estimate for $\lambda_{f}$ at all $\bar{R}$. (This form of the trial wave function was chosen based on the observation that it has a more appropriate asymptotic behavior at large $|\mathbf{r}|$, or $u$.) All results reported below on $\lambda_{f}$ and $I_{f}$ are therefore based on this trial wave function. (cf. Fig. 4 and Table I.) For a variational evaluation of $\lambda_{g}$, we must first put $g=\exp (i \gamma) \bar{g}$ with real $\gamma$ and $\bar{g}$. Equation (17) then becomes

$$
\lambda_{g}=\min _{\gamma, \bar{g}} \frac{\bar{\xi}_{D}^{2} \int\left(\left|\nabla_{\perp} \bar{g}\right|^{2}+\left|\nabla_{\perp} \gamma\right|^{2} \bar{g}^{2}-\left|\nabla_{\perp} \alpha\right|^{2} \bar{g}^{2}\right) d^{2} r-\int\left(\sin ^{2} \alpha\right) \bar{g}^{2} d^{2} r}{\int \bar{g}^{2} d^{2} r} .
$$




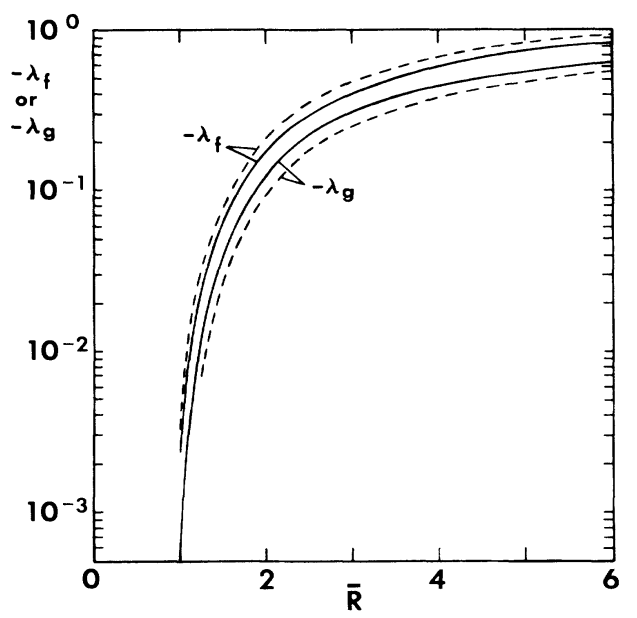

FIG. 4. Present variational results for $\lambda_{f}$ and $\lambda_{g}$ are plotted against $\bar{R}$. The meaning of solid and dashed lines are the same as in Fig. 2.

Varying this expression with respect to $\gamma$ and $\bar{g}$ gives the exact equations

$$
\begin{aligned}
& \nabla_{\perp} \cdot\left(\bar{g}^{2} \nabla_{\perp} \gamma\right)=0, \\
& -\bar{\xi}_{D}^{2}\left(\nabla_{\perp}^{2} \bar{g}+\left|\nabla_{\perp} \alpha\right|^{2} \bar{g}-\left|\nabla_{\perp} \gamma\right|^{2} \bar{g}\right)-\bar{g} \sin ^{2} \alpha=\lambda_{g} \bar{g} .
\end{aligned}
$$

Equation (21) is to be solved with the boundary condition $\gamma \rightarrow \alpha_{\text {sing }}$ as $u \rightarrow 0$ (cf. the Appendix). Clearly the exact solution for $\gamma$ is not $\alpha$. Equation (22) shows that the effective potential for $\bar{g}$ is

$$
V_{\text {eff }}=-\tilde{\xi}_{D}^{2}\left(\left|\nabla_{\perp} \alpha\right|^{2}-\left|\nabla_{\perp} \gamma\right|^{2}\right)-\sin ^{2} \alpha .
$$

Thus if one takes $\gamma= \pm \alpha$ in a variational trial function for $g$, the effective potential would reduce to

$$
V_{\mathrm{eff}}^{(1)}=-\sin ^{2} \alpha,
$$

which is only half as deep as the potential for the longitudinal bound-spin-wave mode. [cf. Eq. (10) or (16)]. Thus with this trial wave function $\left|\lambda_{g}\right|$ would come out much less than $\left|\lambda_{f}\right|$, especially in the weakcoupling limit (i.e., for $\bar{R} \lesssim 1.0$ ), where the eigen"energy" depends exponentially on the negative of the total strength of the potential (cf. Sec. IV for a justification of this point). Now suppose we take $\gamma=\alpha_{\text {sing }}$ of Eq. (2). The effective potential then reduces to

$$
V_{\mathrm{eff}}^{(2)}=-\bar{\xi}_{D}^{2}\left(\left|\nabla_{\perp} \alpha\right|^{2}-\left|\nabla_{\perp} \alpha_{\text {sing }}\right|^{2}\right)-\sin ^{2} \alpha .
$$

Note that $-\widetilde{\xi}_{D}^{2} \int V_{\mathrm{eff}}^{(2)} d^{2} r$ is just what we have minimized in Sec. II with respect to $\alpha(\mathbf{r})$ to obtain the equilibrium $\Delta f$, we thus expect the integrated strength of the first term in Eq. (25) to be comparable in magnitude as that of the second term. In another word, we expect

$$
V_{\mathrm{eff}}^{(2)} \simeq 2 V_{\mathrm{eff}}^{(1)}=V_{\text {long }} \text {. }
$$

Thus the variational estimate for $\lambda_{g}$ obtained with $\gamma=\alpha_{\text {sing }}$ can be quite close to that for $\lambda_{f}$ and therefore represents an enormous improvement over the choice $\gamma=\alpha$, especially in the weak-coupling limit. Of course, according to Eq. (21), neither $\alpha$ nor $\alpha_{\text {sing }}$ is the exact solution for $\gamma$, but we can show (in the next section) that the choice $\gamma=\alpha_{\text {sing }}$ is exact in both the weak-coupling limit $\bar{R} \rightarrow 0$ and the strong-coupling limit $\bar{R} \rightarrow \infty$. Thus the expression

$$
g(\mathbf{r})=e^{i \alpha_{\text {sing }}} f_{2}(u),
$$

with $f_{2}(u)$ given by Eq. (19), should be a good variational trial function for the whole range of $\vec{R}$. In Fig. 4 we have presented our variational result on $\lambda_{g}$ using Eq. (27) as the trial function, as well as our variational result on $\lambda_{f}$ using Eq. (19) as the trial function, for $\bar{R}$ up to 6.0. In Fig. 5 and Table $I$ the reduced satellite intensity $\widetilde{I}_{g}$ calculated with the same trial wave function is presented. The corresponding longitudinal intensity $\widetilde{I}_{f}$

TABLE I. Upper [lower] numbers for each $\bar{R}$ correspond to using $F_{2}$ of Eq. (5b) $\left[F_{1}\right.$ of Eq. (5a)] as the trial function for the function $F(u)$ in Eq. (4). The upper numbers are taken as our predictions because $F_{2}$ gives somewhat lower equilibrium energy than $F_{1}$ for practically the whole range of $\bar{R}$ (cf. Fig. 2). The differences between the upper and lower numbers give some idea on the sensitivity of our variational calculation on the choice of $F(u)$.

\begin{tabular}{cllll}
\hline \hline $\bar{R}$ & \multicolumn{1}{c}{$\lambda_{f}$} & \multicolumn{1}{c}{$\lambda_{g}$} & \multicolumn{1}{c}{$\tilde{I}_{f}$} & $\tilde{I}_{g}$ \\
\hline 1.0 & $-2.44 \times 10^{-3}$ & $-5.7 \times 10^{-4}$ & $1.24 \times 10^{4}$ & $5.81 \times 10^{4}$ \\
& $-3.64 \times 10^{-3}$ & & $0.777 \times 10^{4}$ & \\
1.5 & $-6.41 \times 10^{-2}$ & $-4.06 \times 10^{-2}$ & $2.55 \times 10^{2}$ & $3.25 \times 10^{2}$ \\
& $-7.89 \times 10^{-2}$ & $-2.67 \times 10^{-2}$ & $2.03 \times 10^{2}$ & $5.88 \times 10^{2}$ \\
2.0 & -0.174 & -0.126 & 85.8 & 89.8 \\
& -0.209 & -0.0948 & 72.7 & 140 \\
3.0 & -0.404 & -0.310 & 42.8 & 42.6 \\
& -0.473 & -0.253 & 39.4 & 59.9 \\
4.0 & -0.587 & -0.451 & 36.9 & 36.6 \\
& -0.678 & -0.383 & 35.4 & 49.0 \\
5.0 & -0.725 & -0.553 & 36.9 & 36.5 \\
& -0.830 & -0.481 & 36.2 & 47.5 \\
6.0 & -0.829 & -0.628 & 39.0 & 38.1 \\
& -0.944 & -0.555 & 38.9 & 48.8 \\
\hline \hline
\end{tabular}




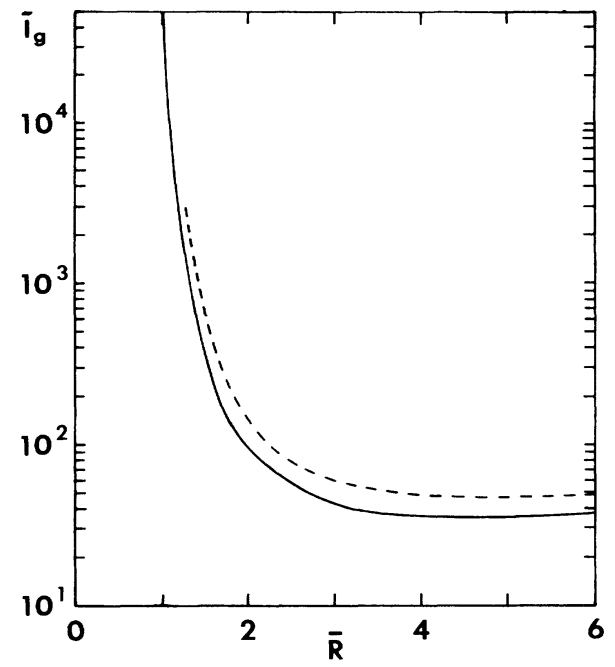

FIG. 5. Present variational result for $\widetilde{I}_{g}$ is plotted against $\bar{R}$, with the same meanings for the solid and dashed lines. $\widetilde{I}_{f}$ is not plotted in the same figure, because it overlaps strongly with $\widetilde{I}_{g}$ (see Table I).

is given in Table I only, and is not plotted in Fig. 5, because the two sets of curves overlap too heavily. We have also found that if $\alpha_{\text {sing }}$ is replaced by $\alpha$ in Eq. (27), then the resulting estimate for $\left|\lambda_{g}\right|$ would be smaller by a factor of 1.83 (or 2.49) at $\bar{R}=6.0$, and by a factor of 16.7 (or 45.4 ) at $\bar{R}=1.5$, depending on whether $F_{1}$ (or $F_{2}$ ) is used to represent the equilibrium texture. Since $\lambda_{g}$ is negative in the whole range of $\bar{R}$, the larger $\left|\lambda_{g}\right|$ obtained with Eq. (27) is clearly the better result. However, we still have some suspicion that the accuracy in our variational results for $\widetilde{I}_{g}$ (and hence $\lambda_{g}$ ?) may not be as good as that for $\widetilde{I}_{f}$ (and $\lambda_{f}$ ?) because the exact ratio $\widetilde{I}_{g} / \widetilde{I}_{f}$ found in the next section for the limit $\bar{R} \rightarrow \infty$ appears to be not borne out by our numerical results up to $\bar{R}=6.0$. It is possible that the eigenenergies are more accurately obtained than the intensities, since the variational approach is a minimum principle for the former only. (The exact asymptotic behaviors $\lambda_{f}$ and $\lambda_{g} \rightarrow 1$ as $\bar{R} \rightarrow \infty$, also found in the next section, do appear to be borne out by our numerical results.)

\section{ASYMPTOTIC ANALYSIS}

We are mostly interested in the limit $\bar{R} \rightarrow \infty$. However, we shall first make a simple discussion of the opposite limit $\bar{R} \rightarrow 0$. In this limit the binding is weak; the wave functions $f$ and $\bar{g}$ are expected to be nearly a constant for a region around the origin much larger than the range of nonvanishing $\alpha$. Equation (21) reduces to $\nabla_{\perp}^{2} \gamma=0$ in this very large region, where $\gamma=\alpha_{\text {sing }}$ is the exact solution. To get a general feeling of the $\vec{R}$ dependence of $\lambda_{f}$ and $\lambda_{g}$ in this limit, we replace their respective potentials by circularly symmetric square-well potentials of strength equal to the values of the corresponding exact potentials at $\mathbf{r}=0$, and their ranges $r_{f}$ and $r_{g}$, respectively, such that

$$
\begin{aligned}
\pi r_{f}^{2} & =\int \sin ^{2} \alpha d^{2} r \equiv(\pi / 2) R^{2} I_{0}(\bar{R}), \\
\pi r_{g}^{2} & =\int\left[\widetilde{\xi}_{D}^{2}\left(\left|\nabla_{\perp} \alpha\right|^{2}-\left|\nabla_{\perp} \alpha_{\text {sing }}\right|^{2}\right)+\sin ^{2} \alpha\right] d^{2} r \\
& =\widetilde{\xi}_{D}^{2} \Delta f(\bar{R}) .
\end{aligned}
$$

In this approximation the Schrödinger equations can be solved exactly, giving

$$
\begin{aligned}
& \lambda_{f}=-\frac{8}{\gamma^{2} \bar{R}^{2} I_{0}(\bar{R})} \exp \left(-\frac{4}{\bar{R}{ }^{2} I_{0}(\bar{R})}\right), \\
& \lambda_{g}=-\frac{4 \pi}{\gamma^{2} \Delta f(\bar{R})} \exp \left[-\frac{4 \pi}{\Delta f(\bar{R})}\right),
\end{aligned}
$$

where $\ln \gamma=0.5772 \ldots$ is the Euler constant. These expressions give the correct exponential $\bar{R}$ dependence of $\lambda_{f}$ and $\lambda_{g}$ in the limit $\bar{R} \rightarrow 0$. However, their front coefficients are not to be trusted quantitatively, since these coefficients depend on the exact shapes of the potentials.

Turning now to the limit $\bar{R} \rightarrow \infty$, we first look at the simpler $\lambda_{f}$ and $I_{f}$. In this limit a $\widehat{\mathbf{d}}$ soliton or domain wall stretches between the two vortex axes. Thus for $x$, $y<R, \alpha$ is a function of $y$ alone, and $F$ of Eq. (6) is minimized by the solution of

$$
d^{2} \alpha / d y^{2}=\widetilde{\xi}_{D}^{-2} \sin \alpha \cos \alpha,
$$

i.e.,

$$
(y /|y|) \sin \alpha=-\widetilde{\xi}_{D} d \alpha / d y=\operatorname{sech}\left(y / \widetilde{\xi}_{D}\right),
$$

where the signs have been chosen to satisfy the boundary conditions that $\alpha \rightarrow 0$ as $y \rightarrow \pm \infty$ and $\alpha \rightarrow \pm \pi / 2$ as $y \rightarrow \pm 0$. Neglecting the $x$ dependence of $f$, Eq. (10) is then solved by

$$
f=\operatorname{sech}\left(y / \widetilde{\xi}_{D}\right), \lambda_{f}=-1,
$$

which also gives

$$
\widetilde{I}_{f} \equiv\left|\int f d^{2} r\right|^{2} / \widetilde{\xi}_{D}^{2} \int f^{2} d^{2} r=\left(\pi^{2} / 2\right) \bar{R} .
$$

Turning now to $\lambda_{g}$ and $I_{g}$, we first notice that

$$
\begin{aligned}
\alpha_{\text {sing }} & \equiv \frac{1}{2}\left(\phi_{1}-\phi_{2}\right) \cong \frac{1}{2}\left\{\left[ \pm \pi-\tan \left(\pi-\phi_{1}\right)\right]-\tan \phi_{2}\right\} \\
& =\frac{1}{2}\left[ \pm \pi-\frac{y}{R / 2-x}-\frac{y}{R / 2+x}\right) \cong \pm \frac{\pi}{2}-\frac{2 y}{R},
\end{aligned}
$$

where the \pm sign stands for $y /|y|$, and

$$
\widetilde{\xi}_{D} \nabla_{\perp} \alpha_{\text {sing }} \cong-2 \widehat{\mathbf{e}}_{y} / \bar{R} \cong 0,
$$

for $x, y \sim \xi_{D} \ll R$ (and $y$ not exactly zero in the last equation). Thus the asymptotic solution of Eq. (21) in this region is simply $\gamma \cong \pm \pi / 2=\alpha_{\text {sing }}$ and $\widetilde{\xi}_{D} \nabla_{\perp} \gamma \cong 0$. Then neglecting the $x$ dependence of $\bar{g}$, and using Eq. (33), we can reduce Eq. (22) to the same equation as that for $f$ in this limit, the solution of which is

$$
\bar{g}=\operatorname{sech}\left(y / \widetilde{\xi}_{D}\right), \lambda_{g}=-1 .
$$

But because Eq. (13) is different from Eq. (12), we find 


$$
\widetilde{I}_{g} \equiv\left|\int g e^{-i \alpha} d^{2} r\right|^{2} / \widetilde{\xi}_{D}^{2} \int|g|^{2} d^{2} r=2 \bar{R} .
$$

Note that had we assumed $\gamma=\alpha$ in this limit, we would be solving Eq. (15). The predicted asymptotic solution would become

$$
\begin{aligned}
& \widetilde{g}=\operatorname{sech}^{v}\left(y / \widetilde{\xi}_{D}\right) \text { with } v=\frac{1}{2}(\sqrt{5}-1), \\
& \lambda_{g}=-v^{2}=-0.3820,
\end{aligned}
$$

and

$\widetilde{I}_{g}=\bar{R}\left\{\left[\Gamma\left[\frac{v}{2}\right]\right]^{4} / 2[\Gamma(v)]^{4} \Gamma(2 v)\right\}=7.29 \bar{R}$.

Equation (41) was given in Ref. 3 as the asymptotic behavior for $\lambda_{g}$ as $\bar{R} \rightarrow \infty$, but we know now that this is incorrect because the assumption $\gamma=\alpha$ is not valid. ${ }^{8}$

There is another point in Ref. 3 that needs to be clarified, namely, the proper reason behind the statement made in Ref. 3 that the physical transverse-spinresonance wave function must have a proper azimuthal symmetry about each axis of $\widehat{\mathbf{d}}$ disgyration. As is briefly mentioned in the Introduction, Ref. 3 cited nonvanishing coupling to a uniform rf field as the reason, while Ref. 4 disagreed and cited a topological reason instead. While the topological point is clearly relevant to half-quantum vortex, it appears inoperative to $\bar{d}$ disgyrations of integer Frank index $m$, so that magnetic coupling argument must be invoked. This leaves the somewhat unsatisfactory situation that two different reasons appear to be needed to justify the same conclusion, depending on whether the Frank index involved is an integer or a half-odd integer. A more unified viewpoint on this matter is clearly desirable, which we have offered in the Appendix. As we shall see there, this new viewpoint can actually give us an unambiguous and general criterion for the determination of the physical transversemagnetic-resonance wave function in the presence of any distribution of $\hat{\mathbf{d}}$ disgyrations of any kinds (assuming a large applied magnetic field).

\section{SUMMARY AND CONCLUSIONS}

In this work we have recalculated the transverse magnetic resonance satellite frequency and intensity associated with a half-quantum vortex pair in a thin slab of ${ }^{3} \mathrm{He}-\boldsymbol{A}$ rotating about the slab normal, and with a magnetic field applied at an angle $\theta$ with the slab normal. This is the geometry considered previously by Salomaa and Volovik for the novel half-quantum vortices to become energetically favorable in the form of vortex "molecules," or bound vortex pairs. However, when analyzing the transverse magnetic resonance satellite frequency and intensity of such a bound pair, they failed to take into account the proper azimuthal symmetry of the spin wave function as is pointed out subsequently by one of us (K.M.). The present calculation uses a variational approach to properly take this into account; thus our results should replace those of Salomaa and Volovik as the predicted magnetic resonance signatures of this novel type of vortices (in the paired state). We find that the transverse satellite resonance shift to be substantially smaller than the value predicted by them, but it is not too small to be totally unobservable. However, one may have to go to high pressure (i.e., near or at the melting pressure), high magnetic field (i.e., $B \gtrsim 0.5 \mathrm{~T}$ ), and low temperature (i.e., $T \lesssim 0.2 T_{c}$ ) in order to observe this satellite resonance. The temperature dependences of this shift and its intensity are also found to be totally different from their predictions. The shift is found to be larger, and the corresponding intensity weaker, as the temperature is lowered, rather than raised. However, some aspects of the original predictions remain correct. The intensity remains proportional to $\Omega$ and $\sin ^{-2} \theta$, and the temperature dependences of the satellite shift and intensity remain very strong, so they can still be used as signatures of this novel type of vortex.

For completeness we have also calculated the corresponding quantities for the longitudinal resonance. We find that the longitudinal resonance actually holds a better promise as a means to observe half-quantum vortices in the paired state, especially if one is not able to perform the experiment at temperatures $T \ll T_{c}$, since in such a case the longitudinal shift $\Delta \omega_{l}$ is already smaller than $\Omega_{L}$ by at least one order of magnitude, whereas the transverse shift is of order $\left(\Delta \omega_{l}\right)^{2} / \gamma H$, which is smaller than $\Delta \omega_{l}$ by a factor $\Delta \omega_{l} / \gamma H$

For the benefit of the experimentalists who might want to perform this experiment, we summarize the following conceptual points. At any given temperature $T$ and pressure $p$ one can determine from published literature $^{6,7}$ the ratio $\rho_{s p}^{\perp} / \rho_{s}^{\perp}$. Then from Ref. 1 and the Fig. 3 of this work one can determine the equilibrium intrapair separation $\bar{R}_{\text {min }}$ (in units of $\xi_{D} / \sin \theta$ ), which depends on $p$ and $T$ via $\rho_{s p}^{\perp} / \rho_{s}^{\perp}$ only. Using this $\bar{R}_{\text {min }}$, one can obtain the values of $\lambda_{f}, \lambda_{g}, \widetilde{I}_{f}$, and $\widetilde{I}_{g}$ from this work (mainly from Table I and Figs. 4 and 5). Then using Eqs. (8), (9), (12), (13), and the definitions of $\widetilde{I}_{f}$ and $\widetilde{I}_{g}$ in Eqs. (35) and (39), one can determine the satellite resonance frequencies and intensities.

Next, we will work out some explicit numbers in order to indicate roughly where one must look for an observable effect. Let us assume that one can observe a $\left|\lambda_{f \text { or } g}\right| \gtrsim 0.1$. Then from Fig. 4 one finds that one must have $\bar{R}_{\text {min }} \gtrsim 2.0$. This requires, from Fig. 3, that $\rho_{s p}^{\perp} / \rho_{s}^{\perp} \lesssim 0.35$. But according to the weakcoupling-plus-Fermi-liquid theory, ${ }^{6}$

$$
\begin{aligned}
& \frac{\rho_{s p}^{\perp}}{\rho_{s}^{\perp}}=\frac{1+\frac{1}{3} F_{1}^{s}\left(\rho_{n o}^{\perp} / \rho\right)}{1+\frac{1}{3} F_{1}^{a}\left(\rho_{n o}^{\perp} / \rho\right)} \frac{1+\frac{1}{3} F_{1}^{a}}{1+\frac{1}{3} F_{1}^{s}}, \\
& \frac{\rho_{n o}^{\perp}}{\rho}(T)=\frac{\left(\frac{\rho_{s p}^{\perp}}{\rho_{s}^{\perp}} F_{1}^{s}-F_{1}^{a}\right)-3\left(1-\frac{\rho_{s p}^{\perp}}{\rho_{s}^{\perp}}\right)}{\left(F_{1}^{s}-\frac{\rho_{s p}^{\perp}}{\rho_{s}^{\perp}} F_{1}^{a}\right)+\frac{1}{3} F_{1}^{s} F_{1}^{a}\left(1-\frac{\rho_{s p}^{\perp}}{\rho_{s}^{\perp}}\right)} .
\end{aligned}
$$

Putting in ${ }^{7} F_{1}^{s}=14.28, F_{1}^{a}=-0.99$, at $P=P_{\text {melting }}$, and $\rho_{s p}^{\perp} / \rho_{s}^{\perp} \lesssim 0.35$, one obtains $\rho_{n o}^{\perp} / \rho \lesssim 0.349$, which roughly indicates $^{6} T \lesssim 0.63 T_{c}$. This estimate shows that the chance of observing the predicted satellite resonances near $T_{c}$ is very small. However, at lower temperatures than $\sim 0.63 T_{c}$, the effects rapidly rise to finite sizes. 
Besides the calculation of the longitudinal and transverse satellite resonance shifts and intensities of a bound pair of half-quantum vortices, this work has also made another contribution, viz., in the Appendix we have obtained a general criterion for the determination of the physical transverse spin resonance wave function for ${ }^{3} \mathrm{He}-A$ in a strong magnetic field in the presence of any distribution of spin disgyrations of any kinds, whether quantized with integer or fractional Frank indices. (The corresponding criterion for the longitudinal resonance is trivial.) This discussion has clarified an issue raised in Ref. 4 about what is the proper justification of the point made in Ref. 3 that the physical spin wave functions for transverse magnetic resonances must have the proper azimuthal symmetry about the axis of each spin disgyration.

Recently Salomaa and Volovik $^{9}$ have proposed that half quantum vortices also play a role in the vortex core transition in ${ }^{3} \mathrm{He}-B$. More specifically, they proposed that the core of the nonaxisymmetric $u$ vortex found in the numerical studies of Thunerberg ${ }^{10}$ and their own ${ }^{11}$ is actually made of two half-quantum vortices confined by a soliton sheet stretched between them, much like quarks are confined inside nucleons and mesons by the gluon strings stretched between the quarks.

While this is theoretically very illuminating, it may be difficult to confirm this proposal experimentally because the situation is complicated by the highly inhomogeneous background order parameter which changes continuously through the following phases as the axes of the half-quantum vortices are approached from afar:

$B$ phase $\rightarrow$ planar phase $\rightarrow$ axiplanar phase $\rightarrow A$ phase.

In this sense the half-quantum vortices studied in this work and in Refs. 1, 3, and 4 are of a much cleaner type with the background order parameter being in the pure $\boldsymbol{A}$ phase with even a uniform $\hat{l}$, allowing more quantitative characterization of these novel objects to be possible. Thus it should remain very interesting to perform the experiments as proposed in Ref. 1 and further investigated in this work, in order to confirm the existence of these novel topological objects. It is hoped that this work has made the conditions of the experiments explicit enough to allow such experiments to be performed successfully. ${ }^{12}$

\section{ACKNOWLEDGMENT}

This work was performed when one of us (C.R.H.) was visiting the University of Southern California (USC). The warm hospitality extended to him by Professors R. Shakeshaft, K. Maki, R. Thompson, and other colleagues at USC is gratefully acknowledged. This research was supported by National Science Foundation under Grant Nos. DMR82-05697 (C.R.H.), DMR8214525, and DMR86-11829 (K.M.).

\section{APPENDIX}

In Ref. 3 one of us (K.M.) noted that the spin wave function $g(\mathbf{r})$ for the physical transverse magnetic resonance mode must have a proper azimuthal symmetry about the axis of any $\hat{\mathrm{d}}$ disgyration, so that the $s$-wave- like solution found in Ref. 1 for a half-quantum vortex pair cannot be physically correct. The reason offered in Ref. 3 is that otherwise the mode cannot couple to a uniform $\mathrm{rf}$ field. In Ref. 4 , the authors of Ref. 1 agreed to the conclusion that their previously found solution is unphysical, but noted a different reason which is topological in character, viz., since the equilibrium textures containing half-quantum vortices are described by doublevalued functions, the corresponding spin wave functions describing the transverse magnetic resonances of such textures must also be double valued.

The purpose of this appendix is to clarify this issue by emphasizing that one always needs to consider both points together in order to properly select the physical solutions without any ambiguity, even though for textures involving only integer- $m$ disgyrations, the implications of the topological point are usually taken for granted.

To elaborate this assertion, we first note that the product $e^{-i \alpha} g$, which appears in Eq. (13), actually has a direct physical interpretation. Namely, it is a principal factor in the resonant part of either of the two transverse components of the spin density (i.e., $S_{x^{\prime} \omega}$ and $S_{y \omega}$ in the choice of coordinates of this work), or, equivalently, of the magnetization. ${ }^{13}$ The remaining factors give the resonant line shape and the separate phase lags of these components, and are independent of the equilibrium texture except via the resonance frequency. As such the product $e^{-i \alpha} g$ must satisfy both of the following two properties.

(i) It must be a single-valued and continuous function of position, since any physical quantity in a macroscopic approach must be single valued and continuous (barring the situation of shock formation which does not concern us here). This point insured that $g$ is single-valued and continuous for any texture containing only integer- $m$ disgyrations - a point that is usually taken for granted! - and $g$ must be double valued and continuous or single valued, but with a sign-changing cut exactly where $\alpha$ has a cut, if the equilibrium texture contains at least one of the half-odd-integer- $m$ disgyrations, since $e^{i \alpha}$ is so in such a case.

(ii) Among the physical choices of $g$ one must further select only those for which $e^{-\alpha} g$ has a nonvanishing space integral, if the magnetic resonance mode as represented by the solution $g$ is to couple to a uniform rf field (which actually only couples directly to $\mathbf{S}_{\omega}$ ), and is also to be detectable by the usual magnetic resonance detectors [which can only detect the (essentially) $k=0$ component of $\mathbf{S}_{\omega}$ ].

Let us now consider some explicit examples. First let us consider an isolated $\widehat{d}$ disgyration of integer Frank index $m$. By symmetry one must have $\alpha=m \phi$. If one writes $g=e^{i \gamma} \bar{g}$ with real $\gamma$ and $\bar{g}$, then point (i) and symmetry imply that $\gamma=m^{\prime} \phi$ with integer $m^{\prime}$, and point (ii) further reduces $m^{\prime}$ to just $m$, because

$$
\begin{aligned}
\left|\int e^{-i \alpha} g d^{2} r\right|^{2}= & \left|\int_{0}^{\infty} \int_{0}^{2 \pi} \cos \left[\left(m^{\prime}-m\right) \phi\right] \bar{g}(r) r d r d \phi\right|^{2} \\
& +\left|\int_{0}^{\infty} \int_{0}^{2 \pi} \sin \left[\left(m^{\prime}-m\right) \phi\right] \bar{g}(r) r d r d \phi\right|^{2},
\end{aligned}
$$

which is nonvanishing only for $m^{\prime}=m$. (Notice that 
even $m^{\prime}=-m$ is excluded!) Next let us consider an isolated $\hat{\mathbf{d}}$ disgyration with $m= \pm \frac{1}{2}$ or $\alpha= \pm \frac{1}{2} \phi$. Point (i) and symmetry now imply that $\gamma / \phi$ must be a half-odd integer, which may always be written as $m^{\prime \prime} \pm \frac{1}{2}$ with integer $m$ ", and point (ii) further reduces $m^{\prime \prime}$ to only zero. Clearly topological reason alone is not sufficient here, since it does not even rule out $\gamma=\mp \frac{1}{2} \phi$. Thus it takes the two conditions together to imply that the phase $\gamma$ of $g$ must increase by exactly $2 m \pi$ when a closed curve surrounding the axis of a $\hat{\mathbf{d}}$ disgyration of Frank index $m$ is traversed in the positive direction, whether $m$ is an integer, or a half-odd-integer. This topological statement remains true when the texture is continuously distorted, so it must remain true when several $\hat{\mathbf{d}}$ disgyrations (of different Frank indices $m_{i}$ in general) are brought into a finite region from infinite separations. In such a case we may replace the above topological statement by a practically more useful boundary condition, viz., if $\left(r_{i}, \phi_{i}\right)$ form the polar coordinates centered around the axis of the $i$ th disgyration line, which is characterized by the Frank index $m_{i}$, then we must have $\gamma \rightarrow m_{i} \phi_{i}$ as $r_{i} \rightarrow 0$. This very precise and general condition for the selection of the physical solutions for $g$ should replace all looser or narrower statements made previously, including those made in Refs. 3 and 4, and is valid whether $m_{i}$ is an integer or a half-odd integer. Note that even though Eq. (13) is real, so that every solution is necessarily degenerate with its own complex conjugate, at most one of such a pair of solutions can be physical. This implies in particular that it is unphysical to look for a solution of Eq. (13) which is real, single valued and continuous, but vanishes where $\alpha$ is discontinuous, even though for such a solution $e^{-i \alpha} g$ would be single valued and continuous. The reason is that such a solution can always be viewed as a linear superposition of a physical $g$ and an unphysical $g^{*}$ which cannot be excited by a uniform $\mathrm{rf}$ field. ${ }^{14}$
${ }^{1}$ M. M. Salomaa and G. E. Volovik, Phys. Rev. Lett. 55, 1184 (1985). Also G. E. Volovik and M. M. Salomaa, Zh. Eksp. Teor. Fiz. 88, 1656 (1985) [Sov. Phys._JETP 61, 986 (1985)].

${ }^{2}$ M. C. Cross and W. F. Brinkman, J. Low. Temp. Phys. 27, 683 (1977); G. E. Volovik and V. P. Mineev, Zh. Eksp. Teor. Fiz. 72, 2256 (1977) [Sov. Phys._JETP 45, 1186 (1977)]; Pis'ma Zh. Eksp. Teor. Fiz. 24, 605 (1976) [JETP Lett. 24, $561(1976)]$

${ }^{3}$ K. Maki, Phys. Rev. Lett. 56, 1312 (1986).

${ }^{4}$ M. M. Salomaa and G. E. Volovik, Phys. Rev. Lett. 56, 1313 (1986).

${ }^{5}$ In searching for such normal mode vibrations, one must consider the "Möbius band" to be rigid in the direction of the $\hat{\mathbf{d}}$ vectors, or else one will obtain normal modes that have nothing to do with this discussion.

${ }^{6}$ R. Combescot, J. Low Temp. Phys. 18, 537 (1975).

${ }^{7}$ D. S. Greywall, Phys. Rev. B 27, 2747 (1983).

${ }^{8} \mathrm{~A}$ preliminary report of this work made during the 1986 APS meeting in Las Vegas, Nevada [Bull. Amer. Phys. Soc. 31, 591 (1986)] contains a number of predictions that are now replaced by new results in this report, because at that time we did not yet realize that $\gamma=\alpha$ is a poor choice for the phase of the spin wave function for the transverse satellite spin resonance.

${ }^{9}$ M. M. Salomaa, ROTA-86 Symposium, Helsinki University of Technology, Espoo, Finland, 1986; M. M. Salomaa and G. E. Volovik, Europhys. Lett. 2, 781 (1986).

${ }^{10}$ E. V. Thuneberg, Phys. Rev. Lett. 56, 359 (1986).

${ }^{11}$ M. M. Salomaa and G. E. Volovik, Phys. Rev. Lett. 56, 363 (1986).

${ }^{12}$ Some readers might wonder whether strong-coupling corrections, which were not incorporated in the analysis of Ref. 1 (or this work), could affect the conclusions of that reference concerning the energetics of the three types of vortices that can exist in ${ }^{3} \mathrm{He}-A$, and thereby render the half-quantum species unstable and therefore unobservable in the situation considered in Ref. 1 and this work. We would like to stress that this is not the case. Unlike in ${ }^{3} \mathrm{He}-B$, where the energies of several kinds of vortices of the same quantum of cir- culation, but of different core structures, were compared (Refs. 10 and 11). Here the three types of vortices are of different quanta of circulation, and therefore their energies are dominated by the flow- and texture-contributions outside their cores, due to the $\ln \left(r_{\Omega} / \xi\right)$ dependences of such contributions. Strong-coupling corrections can only affect these contributions weakly via the small cutoff length $\xi$ for the effective sizes of their cores. Thus strong-coupling corrections are not important in deciding which of these three species of vortices is of the lowest energy in a given situation, as long as $\ln \left(r_{\Omega} / \xi\right) \gg>1$. As $\Omega$ is increased, however, $r_{\Omega}$ decreases. But way before $r_{\Omega}$ becomes as small as $\xi$, it will first become comparable to the intra-pair separation $R$. For such $\Omega$ the analyses of Ref. 1 and this work must already be redone, since the assumption of isolated boundvortex pairs then breaks down. Finally, strong-coupling corrections remain important in determining the core structure of any singular type of vortices, including the halfquantum species.

${ }^{13}$ This and many other fundamental relations given in this work, such as Eqs. (8)-(13), are all derived with the standard spin dynamics as is introduced by A. L. Leggett, Ann. Phys. (N.Y.) 85, 11 (1974); we have thus omitted all of their derivations here. The Leggett approach is based on a Hamiltonian formalism and can be generalized to a space-dependent situation [see, for example, D. J. Bromley, Phys. Rev. B21, 2754 (1980)]. An equivalent Lagrangian formalism has been first developed by one of us [see K. Maki, Phys. Rev. B 11, 4264 (1975); 22, 3493 (1980)]. Note, however, that Eq. (13) was derived in the large external magnetic field limit (i.e., with $\omega \sim \gamma H \gg \Omega_{L}$ ), and under the assumption that the equilibrium texture is confined to the plane $\perp \mathbf{H}$ and is therefore describable by a single angular variable $\alpha$. We must therefore confine our discussion in this appendix to such a situation. How these restrictions can be relaxed remains to be investigated.

${ }^{14}$ This is not in contradiction with the fact that $\delta \widehat{\mathbf{d}}(\mathbf{r}, t)$ is always a real quantity. When solving for a NMR mode the first step is always to write $\delta \hat{\mathbf{d}}(\mathbf{r}, t)$ as $\delta \hat{\mathbf{d}}_{\omega}(\mathbf{r}) e^{-i \omega t}+$ c.c. with a complex $\delta \widehat{\mathbf{d}}_{\omega}(\mathbf{r})$. Clearly the $g(\mathbf{r})$ of Eq. (11) should be 
identified with $\delta \hat{\mathbf{d}}_{\omega}^{\text {(transverse) }}(\mathbf{r})$, with some standard $r$ independent factors removed which give the proper line shape and phase lag of this mode. [cf. also Eq. (7) for how $g(\mathbf{r})$ appears in $\delta \widehat{\mathbf{d}}_{\omega}$.] Note that the minus sign in $e^{-i \alpha}$ of Eq. (13) results directly from assuming the time dependence $e^{-i \omega t}$ for $g$, and changes to a plus sign if the $e^{+i \omega t}$ convention is used. Then the boundary condition $\gamma \rightarrow m_{i} \phi_{i}$ would change to $\gamma \rightarrow-m_{i} \phi_{i}$ as $r_{i} \rightarrow 0$. The most important thing is that one should never invoke both in a single approach, and superposition of these two solutions is absolutely not allowed (i.e., before the $e^{\mp i \omega t}$ factors are restored)! 